\title{
ANALYSIS TECHNIQUESAND PERFORMANCE OF R.C.C POLYMER COMPOSITES
}

\author{
Yash Goyal SM ASCE ${ }^{1}$, Sanju Kumar SM ASCE ${ }^{2}$, Suchismita Sahu ${ }^{3}$ \\ ${ }^{1,2}$ Student, School of Civil Engineering. KIIT University, Bhubaneswar, Odisha, India \\ (Email: yash.goyal108@gmail.com) \\ ${ }^{3}$ Assistant Professor, School of Civil Engineering. KIIT University, Bhubaneswar, Odisha, India \\ (Email: suchismitasahu88@gmail.com)
}

\begin{abstract}
Different compositematerial are widely used in Civil Engineering. The purpose of the present study is to discuss different analysis techniquesfor composite material like Delamination, Generalised differentialQuadrature method and RCC Polymer composite like Fibre Reinforced Polymer and Glass Fibrecomposites. Delamination is subjected to reinforced cement corrosion,in which the volume of the reinforcement of the oxidised metal is greater in volume than the original metal.Generalised differential Quadrature method is an accurate, efficientand simple numerical technique forcomposite structural analysis. Glass Fibrecompositeisa strong light weight material, its bulk strength and weight are also better than many metals. Glass fibreis extensively used for making FRP Tanks and Vessels.Fibre reinforced polymer is a complex made of polymer matrix reinforced with fibres. Glass and carbon are the usual fibres.For any kind of design program which requires weight reduction, exactitudeengineering, finite tolerances, and the clarification of parts in both production and operation, fibre reinforced plastics are the best option.The strengthening systems engagedsignificantly upgrade the flexural as well as shear behaviour of the beams in terms of ductility and failure characteristics. Generalised differentialQuadrature methodproved to have several advantages such as stability, accuracy and easy implementation.
\end{abstract}

Keywords:- Composite Structures, Delamination, FRP based composite, Steel fibre based composite. $* * *$

\section{INTRODUCTION}

FRP composites from the name itself suggests that it is a mixture of two or more materials which, when mixed properly, form a dissimilar material with properties not accessible from the singleconstituents. Fibre reinforced plastic (also fibre - reinforced polymer) is a complex made of a polymer matrix reinforced with fibres. Utilization of FRPs in space engineering, sports and also in automobile industries helped a lot in minimising the price of FRP. The properties of FRP composites and their flexibility leads to higher efficiency, dependability and cost effectiveness in rehabilitation. It can significantly increase the ductility of cementitious materials by improving their resistance to rupture and crack propagation, resulting in increased use of fibre reinforced cementitious composites in recent years, especially the use of structural synthetic polymer fibres.The commonly used fibres are glass, carbon, aramid, or basalt.Carbon fibre-reinforced polymer (CFRP) materials are being widely used in the strengthening of civil engineering reinforced-concrete (RC) structures, either as strips or bars, sheets, mats etc. The usual technique that is used to put CFRP as externally bonded (in general with epoxy) to the exterior of the member to be reinforced $^{1}$. GFRP has the advantages of high strength/weight ratio, corrosion resistance and is also economical comparing over $\mathrm{CFRP}^{2}$. Hence though CFRP is stronger and stiffer, GFRP is used in most of the places because of its expensiveness.
There are different analysis techniques of FRP composites like couple stress theory, layer wise theory, delamination method and Quadrature method. The advantages of the layer wise theory is that it is used to solve the differential equation of various shapes in a closed analytical form ${ }^{3}$. GDQ method can be used to numerically evaluate all the complex problems and the geometric quantities ${ }^{4}$. Couple stress theory can be used for both isotropic and anisotropic materials ${ }^{5}$

Delamination and splitting are typical compressive failure modes of composites with low inter laminar or interfacial shear strength, e.g. carbon fibre - reinforced polymers (CFRPs), and are accompanied by crack propagation, inside the matrix or at the interface, along the loading direction ${ }^{6}$

The purpose of the present study is to discuss different analysis techniques for composite material like Delamination, Generalised different Quadrature method and RCC Polymer composite like Fibre Reinforced Polymer and Glass Fibre composites.

\section{Analysis Techniques of RCC Composites}

\subsection{Layer Wise Theory}

Eisentrageretal3.discussed a finite element formation based on the Layer Wise Theory (LWT) for the test of glass and photovoltaic laminates. For this a user-defined finite element is used inside the Abacus code with the User Element (UEL), where the following problems may be addressed: 
1.The skin layers for laminated glass and photovoltaic structures are modelled by the Kirchhoff plate theory as per LWT. Theorieshaving C0continuity are preferred according to robust finite element formulation. Transverse shear deformation is also included in LWT .

2.Glass and photovoltaic laminates are generally fixed by elastic frames so that the skin layers are partly constrained. LWT uses first-order sheardeformation theory (FSDT) which leads to higher-order system of partial differential equations. Additional degree of freedoms should be regarded in the boundary conditions. Examples are asshown in fig 1(a) and fig 1(b).

Firstly the stress resultant is obtained by integration of the corresponding stresses over the thickness. Principle of virtual work is used for deriving the element stiffness relation based on layer wise theory,using the stiffness relation energy and force matrix is determined and the equation of deflection is also determined 3 .

In Reddy's layerwise laminate plate theory, in every boundary, a set ofunidentified displacement functionsrelating to the inplane width coordinate is established whereas in the depthdirection layerwise linearLagrangian interpolation polynomials are used. The unidentified inplane functions are foundfrom the dominating Euler-Lagrange differential equations. A solution in the form of decaying exponential functions tendsto closed form criticalrepresentations for all free-edge state variables and in the inner laminate regions, the stress state equivalentto classical plate laminate theory 7.

\subsection{Quadrature Method}

Tornabeneet al4. discussed the Quadrature method for composite materials using the Equivalent Single Layer Theory and Layer Wise Theory models for Doubly Curved Shells of elliptic type.

Equivalent SingleLayer Theory

The displacement field equationandthe strain equation is determined for the shell. Thenusing thestress-strain relationship the equation forstress is determined.After that by using several assumptions for doubly curved shell structure and also using Hamilton's principle the equation for Inertia is evaluated and then for solving the differential equations obtained boundary conditions are used such as Clamped edge boundary conditions, Free edge boundary condition and simply supported boundary conditions and the displacement equation for $\tau$ thorder is determined by solving the equations.

Layer WiseModel

In this higher order layer wise theories are used for evaluating the displacement equation for the shell and here forkthlayer it is determined and then by using the straindisplacement equation, equation for strain is determined and also the relation for stress strain is determined, then equation for Inertia is evaluated and the samesteps as done in ESL theory for solving the differential equation are repeated and hence the equation is obtained.

The fundamental equations obtained by equivalent single layer theory and also by usingthe layer wise theory is solved by using the Generalised Differential Quadrature Method and has shown that the GDQ method is less burdensome since no integration is needed, along with GDQ method it uses MATLAB software to solve and to show the deformations4.

Very fewer operations is required in the generalised differential quadrature method for the determination of weighing coefficients of higher order deformation in comparison with the matrix multiplication approach. For solving the buckling issues of plates and also that of columns with interior supports, generalised differential quadrature method is used. Applications of GDQ method is shown in buckling of column and buckling of plates8.

\subsection{Couple Stress Theory}

The couplestress theory is aunusualformat of strain gradient theory, using rotations as variables. The standard couple stress theory was proposed byMindlin,containing only one material characteristic lengthas invariant. In the theory, the strain tensor is similarwhile the curvature tensor is asymmetric.In the paper, a new model without any simplification of new modified couple stress theory is appearedas standard to compare various simplified models, for static bendinginvestigationof compositelaminatedmicroplates based on new modified couple stress theory.

They used variousassumptionsof composite laminated plates of new couple stress theory:

1.Kinematic hypothesis of composite laminated plates:

Classical plate theory is based on kinematichypothesis known as Kirchhoff Hypothesis, according to its hypothesis displacement field is assumed and then the first order shear deformation theory relaxes on one of the condition of theKirchhoff Hypothesis, so accordingly it gives its own displacement field according to its conditions, then the higher order shear deformation theory relaxes on one of the condition of the Kirchhoff hypothesis. So Reddy proposed a theory keeping higher order shear deformation theory in mind and then he gave his displacement field.

\section{Fundamental hypothesis of New Modified Couple Stress Theory:}

In the plate couple stress theory $\omega$ is the rotation, while in the modified couple stress theory win the z-direction was assumed to be zero for laminated composite plate model, In Kirchhoff Hypothesis the rotation $\omega$ in the z-direction is neglected then the strain and bend tensor in the composite Reddy theory was determined.

As previous study was made with some simplification that is $(\omega z=0, \varkappa x z=0, x y z=0)$ and

$(\omega z=0, \varkappa x z \neq 0, \varkappa y z \neq 0)$ but the present study is done without any simplification that is $(\omega z \neq 0, x x z \neq 0, x y z \neq 0)$ then the stress-strain relation, elastic stiffness matrix and the displacement equation was determined5.

\subsection{Delamination Method}

When reinforced concrete structuresexperiences a reinforcement corrosion, a delamination occurs in them. Delamination is a mode of failure for composite materials. During this failure, the volume of the 
original metals exceed when the metal oxidises. Hence, a large amount of space is used for the oxidised metals. Hence, the weaker tensile strength of concrete is overcome by a wedge like stress on the concrete which creates a partition between the concretes of the reinforcing bars.

Rabinovitch and Frostigpresented a reliable and a model that foretell thebeginning and increase of the interfacial delamination at the border of the FRP strip and in the area of flexural and shear cracks.

It isdoneall the way through in two phases:

(1) "Stress Analysis" where the stress and deformation fields of the member are established.

(2) "Fracture Mechanics" where an energetic failure criterion is determined.

\section{Stress Analysis}

Thearithmetical formulation for the retrofitted beamdepends on thevariational principle of potential energy minimization and the idea of closed form high order theory.

Here the equilibrium equations obtained using thevariational principle and the hypothesisof small deformations to obtain the generalised constitutiverelationships for the RC beam and theFRP strips. Then the closed form solution using kinematic relationshipof smalldeformations and Hooke's Law is obtained. Thedissimilarityamong the fully bonded part, the delaminated partwith contact, and the delaminated part without contact which is based on the structuralresponse and the delamination increase.In thearithmetical formation, the continuity conditions have two major aspects. The primary one is to merge and to connect the different parts of the strengthened beam. The nextaspect is to figure outthe flexural and shear cracks in the RC member. Then the set of continuity circumstances at the flexural crack section, the crack due to rotational discontinuity, the stresses and the stress resultants at eachpoint of the beam is determined .

Fracture Analysis

The interfacial delamination and its growth rate is attained by acquiring the energetic criterion of the elastic energy release rate(ERR). Then the $\mathrm{J}$-integralis determined which is based on ERR. After thatthestrain energy density and the deformation was determined 9 .

Yamanaka et al10. studied the delamination growth in a fabric composite using a multiscale finite element analysis using ANSYS 12.0 in a double cantilever beam (DCB) specimen. The R-curvesand the load-displacement curves obtained from this analysis agree with the lower bound of the experimental results 10 .

\section{RCC composite}

$\mathrm{RCC}$ is weak in tension and moderate in compression. To improve its strength and durability several composite materials are used such as FRP, GFRP, CFRP.

\subsection{FRP}

Fibre reinforced polymer (FRP) composites that have high strength fibres which are embedded in a matrix of polymer resin as in fig.2.

FRPs are lighter, durable and possess high tensile strength, which provides an excellent material to bear the tensile strengthen RC structure in flexure as well as in shear11,12.FRPs are used for retrofitting of different concrete structures like beams, columns, slabs and walls. Typical values for properties of the fibres are given in Table 1.

FRPs also have higher strength-to-weight ratio as compared to traditional material such as steel, which leads to requirement to less labour and equipment intensive and the time required for retrofitting is shortened12.FRP increases the ductility of cementitious materials by improving the resistance to rupture and crack propagation due to which fibre reinforced concrete has been used to concrete structures including shotcrete, tunnel lining, maritime structures, seismic structures and slab on grade12,13.The FRP composites have compressive material properties sensitive to temperature elevations. These usually occur in application of engineering (i.e in hot environment or incidents involving fire occur). Due to these reasons, failure occur at glass state, kinking failure at the glass transitional bulking with subsequent post-peak kinking at rubbery state6.

\subsection{CFRP}

CFRP (as shown in fig.3) materials have high strength, light weight, good resistance against chemicals and good fatigue strength that makes it an ideal material to be used in engineering works like bridges repairs and rehabilitation 14 as shown in fig.4. However due to the polymeric nature of CFRP matrix and of the epoxy adhesive, its behaviour at higher temperature is a cause of concern 11 .

CFRP materials are bonded with structural member with the help of adhesive which in turn increases the strength and stiffness of the member to be strengthened 1 . The main advantage of using such a method is because it is easy to apply and special anchorages are eliminated used in post tensioning method. This indicates enhancement in concrete confinement as a result of using CFRP.

\section{GFRP}

Due to low cost of Glass fibre-reinforced polymer(GFRP) bars these are used in concrete bridge deck slabs and other structure of concrete 15 .

Most commonly used reinforcement are represented by glass fibre (as shown in fig.5) due to their properties like sound absorption, thermal and electrical insulation corrosion resistance and low cost compared to other types of fibres like carbon. Glass fibres has lots of applications for examples in car bodies, electronic devices, aircraft parts and many other components of 
our daily life use16. Retrofitting of columns also use glass fibres as shown in fig.611. Composite materials are more advantageous than metals as it has high specific strength and stiffness as well as reduction in weight. Fibre composite may delaminate during their lifetime because of their low intra-laminar strength16.

Seismic - resistant structures use Buckling restraint braces(BRBs) where glass fibre-reinforced polymer (GFRP) steel BRB is proposed a suitable material. The external restraining components use GFRP wrapping layer and GFRP pultruded tube for wrapping of steel structure. The novel GFRP steel BRB is easily transported, easy to lift and install as it is lightweight. The main part of GFRP pultruded tube is shown in fig.7.

The local stability of the BRB can be improved with the help of a core steel brace which has cruciform cross section and also a mortar filled GFRP tube. For practical engineering, the GFRP steel BRB which has cruciform cross section is recommended. The GFRP steel BRB core steel brace is capable of better local stability 16

\section{DISCUSSION}

Analysis Technique:

As discussed above in the analysis techniques, LWT has used Finite Element Method, where shear deformation has been included. Also, LWT solves the problem of higher order partial differential equations3.Euler's equations are introduced to solve layer wise theory7. In GDQ method, MATLAB software is used to solve and to show the deformations of different geometric shapes. Also application of this method is shown in buckling of columns and plates. In couple stress theory, Static bonding analysis of composite laminates plates has been done. Delamination which is a failure mode of composite materials where crack failure begins at the edge of FRP strip.

Composite polymer:

For improving the performance of RCC, FPRs are used because of its several advantages.FRPs are used for retrofitting and rehabilitation purpose in different concrete structures. FRP strengthens the RC structure in shear as well as in Flexure by delaying the crack formation and rupture.Most common method is to apply FRP as externally bonded with epoxy to the surface of member to be strengthened11.GFRP has also its application in seismic restraint structures used as Buckling-Restraint braces (BRBs)16.

\section{CONCLUSION}

Among the four method discussed, GDQ method is more efficient and less burdensome as it has no integration. This method is less time consuming as it uses software like MATLAB. This method is also applicable for buckling problem. Due to its high accuracy and convenience this method is widely used in structural analysis.Mechanical properties of CFRP is more than GFRP. One of the main disadvantages of
CFRP is that it is costlier than GFRP. Demerits of FRP is that it is very sensitive to temperature.

\section{REFERENCES-}

[1] by J. P. Firmo1; J. R. Correia2; D. Pitta3; C. Tiago4; and M. R. T. Arruda5. "Bond Behavior between Near - Surface - Mounted CFRP Strips and Concrete at High Temperatures": 2014.

[2] Advances in building technology, part 2002, page811.

[3] J. Eisenträger , K. Naumenko , H. Altenbach , J. Meenen." A user defined finite for laminated glass panel and photovoltaic modules based on a layer wise theory ":2015

[4] FrancescoTornabene, Nicholas Fantuzzi, Michele Bacciocchi, Rossana Dimitri . "Dynamic analysis of thick and thin elliptic shell structures made of laminated composite materials":2015.

[5] ShengqiYang ,Wanji Chen. "On hypotheses of composite laminated plates on new modified couple stress theory":2015.

[6] Wei Sun, Anastasios P. Vassilopoulos, Thomas Keller). "Effect of temperature on kinking failure mode of nonslender glass fiber reinforced polymer specimens":2015.

[7] C. Mittelstedt,W. Becker Reddy's layerwise laminate plate theory for the computation of elastic fields in the vicinity of straight free laminate edges" 2007.

[8] H. Du; K. M. Liew/ and M. K. Lim , "Generalized differential Quadrature method for buckling analysis".

[9] O. Rabinovitch1 and Y. Frostig "Delamination of RC beams strengthened with FRP strips - A closed Form High Order and Fracture Mechanics Approach".

[10] Tadayoshi Yamanaka , Hossein Ghiasi , Mohammad Heidari-Rarani , Larry Lessard, Victor Feret, Pascal Hubert "Multiscale finite element analysis of mode I delamination growthin a fabric composite":2015.

[11](YASMEEN TALEB OBAIDAT) "Structural Retrofitting of reinforced concrete beams usingCFRP":2010.

[12] byShamim A. Sheikh, David DeRose, and Jamil Mardukhi. "Retrofitting of Concrete Structures for Shear and Flexure with Fiber-Reinforced".

[13](Huy Pham, Riadh Al-Mahaidi) "Experimental investigation into flexural retrofitting of reinforced concrete bridge beams using FRP composites":2004.

[14] M.A Shahawy, $M$ Acrokiasamy, $T$ Beitelman and $R$ Sowrirajan. "Reinforced concrete rectangular beams strengthened with CFRP laminates":1995.

[15] CarosenaMeola $\Uparrow$, Giovanni M. Carlomagno)."Impact damage in GFRP: New insights with infrared thermography":2010.

[16] 16. by Kailai Deng ; Peng Pan ; XinNie; XiaoguangXu ; Peng Feng ; and Lieping Ye "Study of GFRP Steel Buckling Restraint Braces":2015. 


\section{FIGURE -}

\subsection{LAYER WISE THEORY-}

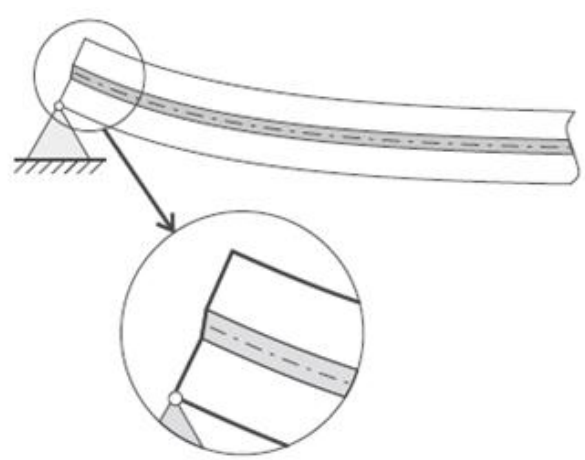

Fig. 1. (a) Deformation of plate support without rotational constraint ${ }^{3}$

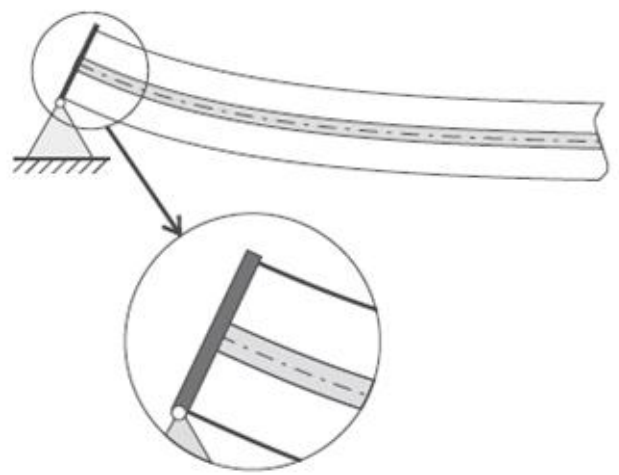

Fig. 1. (b) Deformation of plate support with rotational constraint ${ }^{3}$.

TABLE.1 Strength and stiffness of component used ${ }^{11}$

\begin{tabular}{ccccc}
\hline Material & $\begin{array}{c}\text { Tensile strength } \\
(\mathbf{M P a})\end{array}$ & $\begin{array}{c}\text { Modulus of elasticity } \\
(\mathbf{G P a})\end{array}$ & $\begin{array}{c}\text { Density } \\
\left(\mathbf{k g} / \mathbf{m}^{\mathbf{3}}\right)\end{array}$ & $\begin{array}{c}\text { Modulus of elasticity to } \\
\text { density } \mathbf{~ r a t i o ~}\left(\mathbf{M m}^{\mathbf{2}} / \mathbf{s}^{\mathbf{2}}\right)\end{array}$ \\
\hline Carbon & $2200-5600$ & $240-830$ & $1800-2200$ & $130-380$ \\
Aramid & $2400-3600$ & $130-160$ & $1400-1500$ & $90-110$ \\
Glass & $3400-4800$ & $70-90$ & $2200-2500$ & $31-33$ \\
Epoxy & 60 & 2.5 & $1100-1400$ & $1.8-2.3$ \\
CFRP & $1500-3700$ & $160-540$ & $1400-1700$ & $110-320$ \\
Steel & $280-1900$ & $190-210$ & 7900 & $24-27$ \\
\hline
\end{tabular}

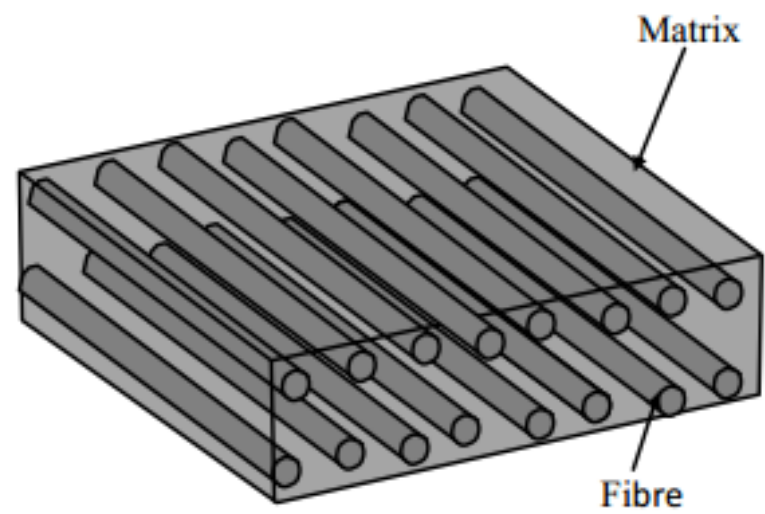

fig.2. A systematic diagram shows a typical unidirectional FRP plate ${ }^{11}$ 


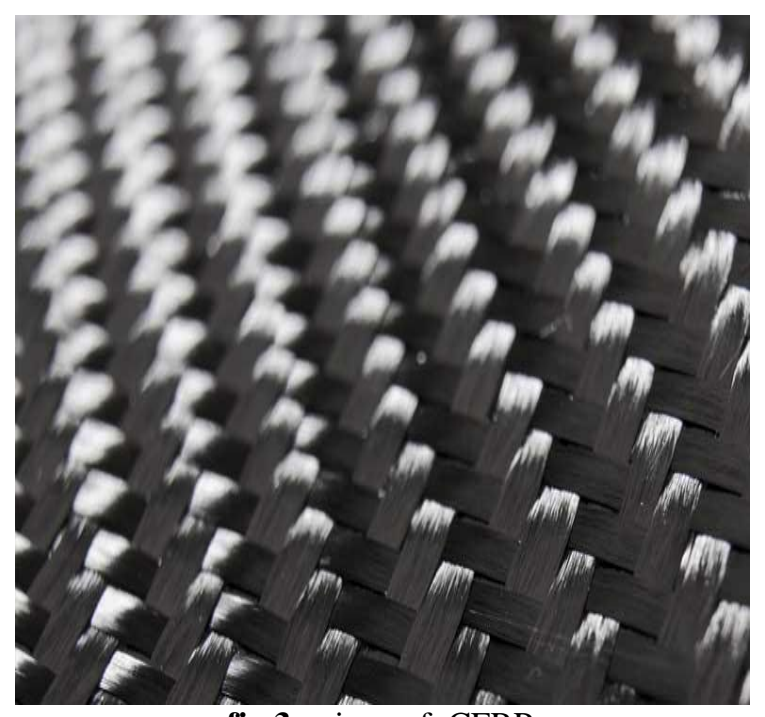

fig.3. view of CFRP

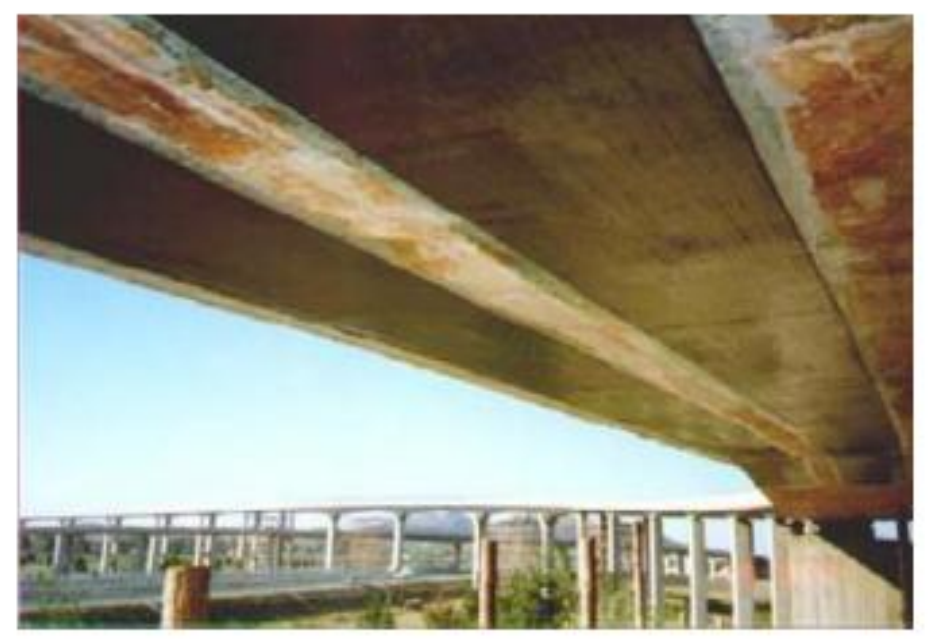

fig.4. strengthening of a highway RC bridge slab in China ${ }^{11}$.

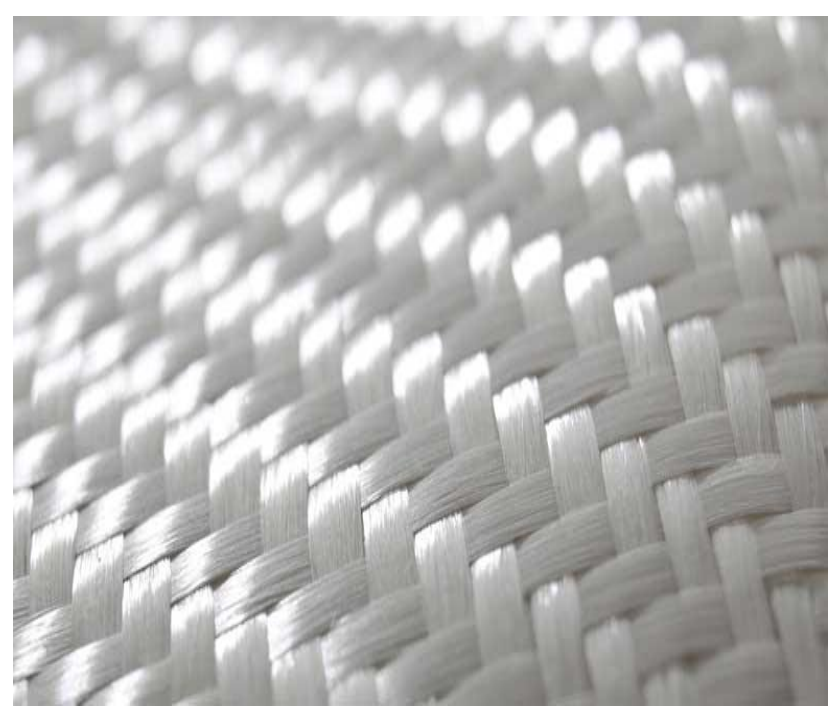

fig.5. view of GFRP 


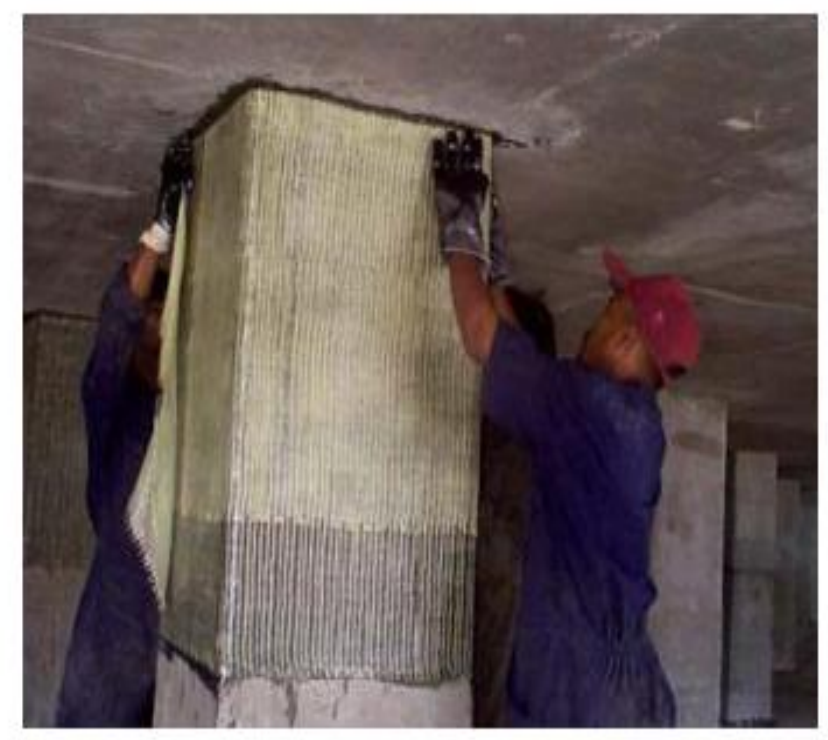

fig.6- retrofit of columns for a cryogenic tank in Gujarat, India ${ }^{11}$.

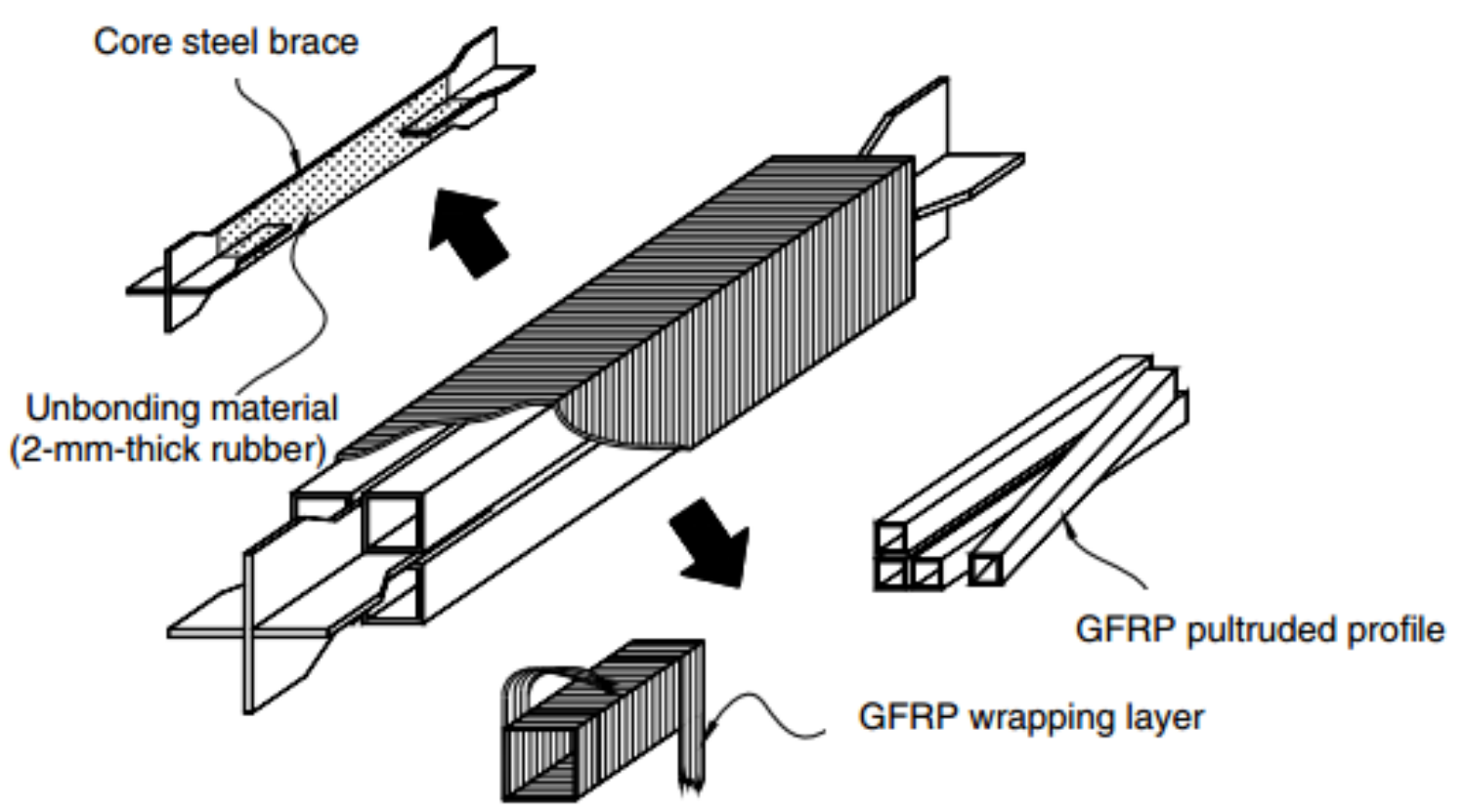

fig.7.Proposed GFRP steel BRB ${ }^{16}$ 